\title{
Nasal Flaring
}

National Cancer Institute

\section{Source}

National Cancer Institute. Nasal Flaring. NCI Thesaurus. Code C87099.

Enlargement of the nostrils during breathing; although there are many possible causes, it can be a sign of respiratory distress in young infants. 